\title{
Long-term efficacy and safety of letrozole for the adjuvant treatment of early breast cancer in postmenopausal women: a review
}

This article was published in the following Dove Press journal:

Therapeutics and Clinical Risk Management

10 September 2009

Number of times this article has been viewed

\begin{abstract}
Alain Monnier
Institut Régional Fédératif du Cancer (IFRC), Centre Hospitalier BelfortMontbéliard, Montbéliard, France
\end{abstract}

\begin{abstract}
Aromatase inhibitors (AIs) are becoming more widely used than tamoxifen as adjuvant hormonal therapy for postmenopausal women (PMW) with early breast cancer (EBC). It is clear that these drugs offer important efficacy benefits over tamoxifen and differ from tamoxifen in their safety profile. The accepted strategies for adjuvant AI therapy include initial adjuvant treatment following surgery, switching and/or sequencing from prior tamoxifen, and extended adjuvant therapy following the full 5 years of tamoxifen treatment. Among the available AIs, letrozole has been evaluated in large, well-controlled, double-blind clinical trials in the initial adjuvant, extended adjuvant, and more recently, the sequential adjuvant settings. Letrozole is the most potent of the AIs and provides near complete suppression of plasma estrogens in PMW. Letrozole also significantly reduces the occurrence of early distant metastases, the most lethal type of recurrence event, which can lead to improved survival. Clinical comparisons of letrozole with both tamoxifen and placebo have also provided important long-term safety data on the use of AIs as adjuvant therapy in PMW with EBC. The weight of clinical evidence indicates that letrozole is a safe and effective option for adjuvant hormonal therapy across all three AI treatment settings.
\end{abstract}

Keywords: aromatase inhibitor, breast cancer, hormonal therapy, letrozole, postmenopausal women, tamoxifen

\section{Introduction}

While adjuvant hormonal therapy continues to be the standard of care for postmenopausal women (PMW) with early breast cancer (EBC), there has been a shift in the treatment paradigm away from the more traditional 5 years of adjuvant tamoxifen to strategies employing the third-generation aromatase inhibitors (AIs) letrozole, anastrozole, and exemestane. A recent study found a marked increase in the use of AIs between the years 2000 and 2003, while the use of tamoxifen declined over the same time (Figure 1). ${ }^{1}$ In clinical trials, AIs have proven superior to tamoxifen in reducing breast cancer recurrence, although the treatment strategies examined have not been uniform across trials. While the Breast International Group (BIG) $1-98^{2-5}$ and the Arimidex Tamoxifen Alone or in Combination (ATAC) ${ }^{6,7}$ trials have proven the superiority of the nonsteroidal AIs (letrozole or anastrozole, respectively) as initial adjuvant therapy compared with 5 years of tamoxifen, the Intergroup Exemestane Study (IES) has shown the superiority of switching to the steroidal AI exemestane following 2 to 3 years of prior tamoxifen, in comparison with continuing tamoxifen. ${ }^{8,9}$ Other trials, including the Arimidex Nolvadex (ARNO) 95 study and the Italian Tamoxifen Arimidex (ITA) trial have examined a similar switch strategy using anastrozole. ${ }^{10,11}$ 
Al Dispensing within 2 years of diagnosis

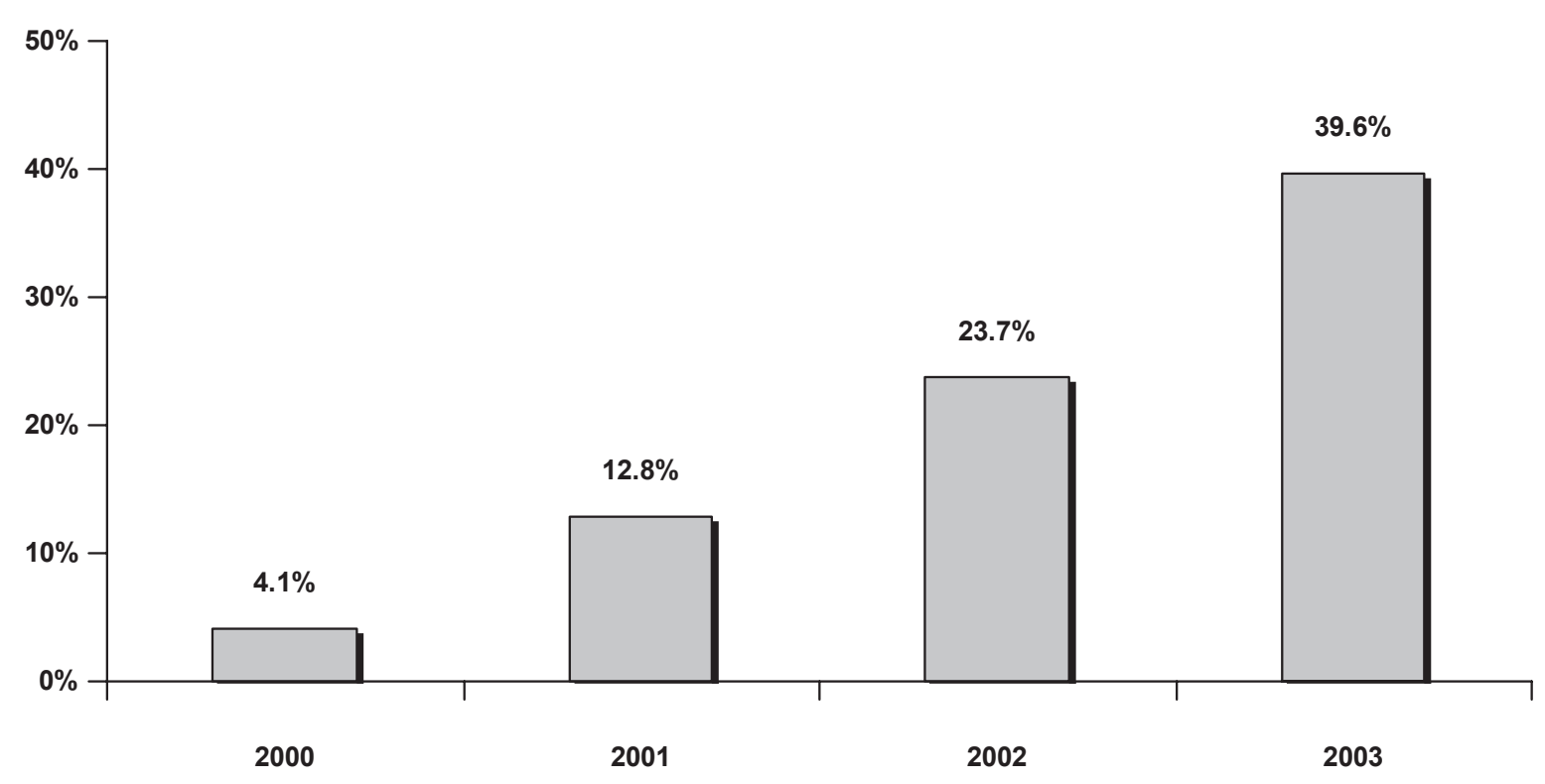

Figure I Dispensing of aromatase inhibitors within 2 years of diagnosis among women $>55$ years old $(N=13,245)$ diagnosed with hormone receptor-positive breast cancer, enrolled at 7 integrated health care delivery systems in the Cancer Research Network, for calendar years 2000-2003. Drawn from data of Hortobagyi et al $2004{ }^{26}$

BIG 1-98 also allowed for an analysis of sequential adjuvant therapy with letrozole before or after tamoxifen compared with letrozole monotherapy., ${ }^{2,5}$ The MA.17 trial has also examined the efficacy and safety of letrozole following the standard 5 years of adjuvant tamoxifen, in comparison with placebo (extended adjuvant therapy). ${ }^{12,13}$ The AIs have important safety benefits relative to tamoxifen. Whereas the adverse event (AE) profile associated with AI treatment is largely predictable in women undergoing extreme estrogen deprivation, sporadic and/or unforeseen serious complications such as stroke, thromboembolism, and endometrial cancer can occur with tamoxifen. ${ }^{14,15}$ Although the findings of the major AI trials support the use of AIs in these different treatment settings, the weight of clinical evidence strongly supports the initial use of AIs, while a switch to an AI after tamoxifen is an option for patients who do not begin their adjuvant treatment with an $\mathrm{AI}$.

Letrozole is somewhat unique in that it has been evaluated in large, randomized, well-controlled trials across all three of the treatment paradigms (ie, initial adjuvant, sequential adjuvant, and extended adjuvant). As BIG 1-98 was initiated at a later time than trials such as ATAC or IES, knowledge of the overall AI side-effect profile also allowed for a more thorough assessment of its safety. Letrozole is effective in suppressing plasma estrogen levels to near-undetectable levels in PMW with EBC. ${ }^{16-18}$ Initial adjuvant letrozole has also proven significantly more effective than tamoxifen in reducing early distant metastases (DM), ${ }^{2,19}$ and this finding may be especially pertinent given the natural history of breast cancer. DM are known to be the most lethal of all recurrence events, ${ }^{20}$ and recent data demonstrate there is an early peak of recurrence at 2 years, most of which are DM recurrences, in patients receiving tamoxifen. ${ }^{21}$ It is therefore likely that treatments that reduce DM will eventually prolong overall survival (OS), and recent results with letrozole are supportive of this contention. ${ }^{3,5}$ In this review, we examine the efficacy and safety profile of letrozole when used as adjuvant therapy in PMW with EBC. Available efficacy and safety data from both published and abstract sources regarding the use of letrozole in the initial adjuvant, sequential adjuvant, and extended adjuvant settings were reviewed, as were studies with other AIs, where relevant, for comparative purposes.

\section{Efficacy of letrozole Initial adjuvant therapy}

BIG 1-98 was a uniquely designed trial initially developed to evaluate the impact of 5 years of letrozole or tamoxifen as initial adjuvant therapy in PMW with hormone receptorpositive $(\mathrm{HR}+) \mathrm{EBC}$; it was later amended to include two sequential treatment arms, with letrozole or tamoxifen for the first 2 years, followed by the other drug for the remaining 3 years of adjuvant treatment. ${ }^{2}$ The rigorous trial design also 
provided for a large central assessment of receptor status, comprehensive safety assessment using Common Toxicity Criteria (CTC) 2.0 criteria, and lifelong monitoring of cardiovascular events; the International Breast Cancer Study Group (IBCSG), an independent academic research group, conducted the trial. The primary core analysis (PCA) compared initial therapy with letrozole and tamoxifen, including patients randomly assigned to sequential treatment, with all events censored after 30 days following treatment switch $(\mathrm{N}=8,010)$. Later analyses at 5 and 10 years after initiation were planned for women assigned to the monotherapy arms (letrozole or tamoxifen for 5 years). ${ }^{2}$ The initial analysis, at a median 25.8-month follow-up, reported a significant 19\% improvement in disease-free survival (DFS), the primary end point, a significant $28 \%$ improvement in time to recurrence, and a significant $27 \%$ improvement in time to distant recurrence (TTDR), as well as a trend toward improved OS compared with patients on tamoxifen (Table 1). Prospectively-planned subgroup analysis also showed letrozole to be significantly more effective than tamoxifen across important patient groups, such as those with node-positive $(\mathrm{N}+)$ disease (hazard ratio [HR] $0.71 ; P<0.001$ ), and those with large primary tumors (HR $0.76 ; P=0.004$ ). ${ }^{2}$

Following the reporting of these pivotal results, particularly in view of the significant DFS advantage and DM reductions with letrozole, the Data Safety Monitoring Board recommended that the IBCSG unblind the tamoxifen arm for ethical reasons. The IBCSG decided to counsel patients in the tamoxifen arm regarding letrozole's superiority, and allow them to electively cross over to letrozole, while leaving the remaining arms blinded. ${ }^{3,5}$ Subsequent analyses are therefore impacted by the crossover of 619 patients $(25.2 \%)$ from the tamoxifen arm. Notably, most patients who crossed over did so during years 3 to 5 and were generally high-risk patients, more likely to have $\mathrm{N}+$ disease and larger tumors, vs those electing not to cross over. ${ }^{3,5}$ Despite the crossover in the intent-to-treat (ITT) population, long-term follow-up (median, 76 months) of the monotherapy population $(\mathrm{N}=4,922)$ showed a $12 \%$ improvement in DFS, a $15 \%$ improvement in TTDR, and a $13 \%$ improvement in OS that is approaching significance with letrozole relative to placebo in the ITT population (Table 1). Because of the crossover, the true benefit of letrozole therapy is likely not reflected in the ITT analysis. An additional analysis has been reported that censors patients enrolled into the tamoxifen arm who elected to receive letrozole at the time of crossover, in an attempt to correct for the dilution of the letrozole effect. The censored analysis showed an even greater and significant superiority of letrozole on all end points, including OS, with a $16 \%$ improvement in DFS, a $19 \%$ improvement in TTDR, and a 19\% improvement in OS (Table 1). ${ }^{5}$ Although both the ITT and censored results are subject to potential biases, in favor of tamoxifen or letrozole, the true letrozole benefit over tamoxifen likely lies between the two. Recently reported findings at a median 60.5-month follow-up of the PCA population ( $\mathrm{N}=8,010)$ have corroborated these findings in both the ITT and censored analyses. ${ }^{3}$ These results support the hypothesis that the early profound reduction in DM with letrozole leads to a survival benefit with longer follow-up.

The findings of BIG 1-98 demonstrate the superiority of letrozole over tamoxifen as initial adjuvant therapy in reducing recurrences overall, as well as DM recurrences, and the end point of OS has consistently trended better. Recent evidence indicates that DM are the most common type of

Table I Efficacy end points from the Breast International Group (BIG) I-98 and MA.I7 trials: hazard ratios (HR) and (P value) for disease-free survival (DFS), time to recurrence (TTR), time to distant recurrence (TTDR), distant disease-free survival (DDFS), and overall survival $(\mathrm{OS})^{2,5,13}$

\begin{tabular}{lllll}
\hline & BIG I-98 & & MA.I7 \\
\cline { 2 - 3 } & Letrozole vs tamoxifen & & Letrozole vs placebo \\
\hline Follow-up & $25.8 \mathrm{mo}$ & $76.0 \mathrm{mo}^{\mathrm{a}}$ & $30.0 \mathrm{mo}$ \\
$\mathrm{N}$ & 8,010 & 4,922 & 5,187 \\
DFS (P value) & $0.8 \mathrm{I}(0.003)$ & $0.88(0.03) 0.84^{\mathrm{CEN}}(0.74-0.95)$ & $0.58(<0.00 \mathrm{I})$ \\
TTR $(P$ value $)$ & $0.72(<0.00 \mathrm{I})$ & $\mathrm{NR}$ & $\mathrm{NR}$ \\
TTDR $(P$ value $)$ & $0.73(0.00 \mathrm{I})$ & $0.85(0.05) 0.8 \mathrm{I}^{\mathrm{CEN}}(0.68-0.96)$ & $\mathrm{NR}$ \\
DDFS $(P$ value $)$ & $\mathrm{NR}$ & $\mathrm{NR}$ & $0.60(0.002)$ \\
OS $(P$ value $)$ & $0.86(0.16)$ & $0.87(0.08) 0.8 \mathrm{I}^{\mathrm{CEN}}(0.69-0.94)$ & $0.82(0.3)$ \\
\hline
\end{tabular}

a HR for the intent-to-treat (ITT) analysis of the monotherapy arms at 76 months ( $P$ value) is shown; the HR ( $\mathrm{RR}^{\mathrm{CEN}}$ ) for the censored analysis (with $95 \%$ confidence interval) is shown below the ITT value. Abbreviation: NR, not reported. 
early recurrence event (occurring within 2 years of surgery) in patients on tamoxifen, ${ }^{21}$ and the benefit of letrozole on DM may be especially relevant, as distant recurrences have been associated with reduced OS and death from breast cancer compared with local recurrences. ${ }^{20}$ A prospectively planned retrospective analysis of BIG 1-98, which focused on early recurrence events (at 2 years), reported DM recurrences to be the predominant recurrence event at this time point, accounting for $74 \%$ of all recurrences. ${ }^{19}$ Letrozole significantly reduced early recurrence risk by $31 \%$ over tamoxifen in this analysis ( 117 events vs 168 events, HR $0.69 ; P=0.002$ ), with a $30 \%$ absolute reduction in DM events ( 87 vs 125 events, $2.3 \%$ vs 3.3\%) (Figure 2). ${ }^{19}$ Retrospective analysis from the similarly designed ATAC trial showed only a 7\% reduction in early (2.5 years) DM events with anastrozole over tamoxifen. ${ }^{22}$ Notably, a significant effect of anastrozole on DM recurrences among the $\mathrm{HR}+$ patients $(\mathrm{N}=5,216)$ was not observed until after treatment completion, at a median follow-up of 100 months; no improvement in OS has emerged in the ATAC trial, and 5 fewer overall deaths were seen with anastrozole treatment relative to tamoxifen (all cause deaths, 472 vs 477; HR 0.97; $P=0.7){ }^{7}$ In contrast, at 76 months in BIG 1-98 $(\mathrm{N}=4,922)$, there were 40 fewer deaths with letrozole (overall deaths 303 vs 343), and this difference was due to the avoidance of cancer death events, as the number of deaths without a prior cancer event was equal in the two arms (87 and 87 events). ${ }^{5}$ The findings of BIG 1-98 over time show the importance of reducing early DM events and support the contention that significant reductions in early DM will lead to long-term improvements in OS. ${ }^{23}$

\section{Letrozole vs anastrozole - FACE}

The difference in outcome between ATAC and BIG 1-98, as noted above, is illustrative of the impact of early DM reduction on survival and also suggests a potential difference in efficacy between these two nonsteroidal AIs. Indeed, there is evidence for a greater suppression of both plasma and tissue estrogen levels with letrozole compared with anastrozole treatment (Figure 3). ${ }^{16-18}$ While ATAC and BIG 1-98 are not directly comparable because of differences in design and follow-up, a recently completed trial, the Femara Anastrozole Clinical Evaluation (FACE), has compared the efficacy and safety of initial adjuvant treatment with these agents in a head-to-head fashion in a population of PMW with $\mathrm{HR}+, \mathrm{N}+, \mathrm{EBC} .{ }^{24,25}$ The forthcoming results of this trial, once available, should provide, for the first time, a directly comparative assessment of both efficacy and safety for these two AIs in the important high-risk population of $\mathrm{N}+\mathrm{EBC}$ patients.

\section{Sequential adjuvant therapy - BIG I-98}

The sequential arms of BIG 1-98 were designed to evaluate the efficacy and safety of letrozole and tamoxifen used in sequence with either agent for the first 2 years followed by the other for the remaining 3 years (letrozole $\rightarrow$ tamoxifen or tamoxifen $\rightarrow$ letrozole). Although other trials (eg, IES,

Tamoxifen

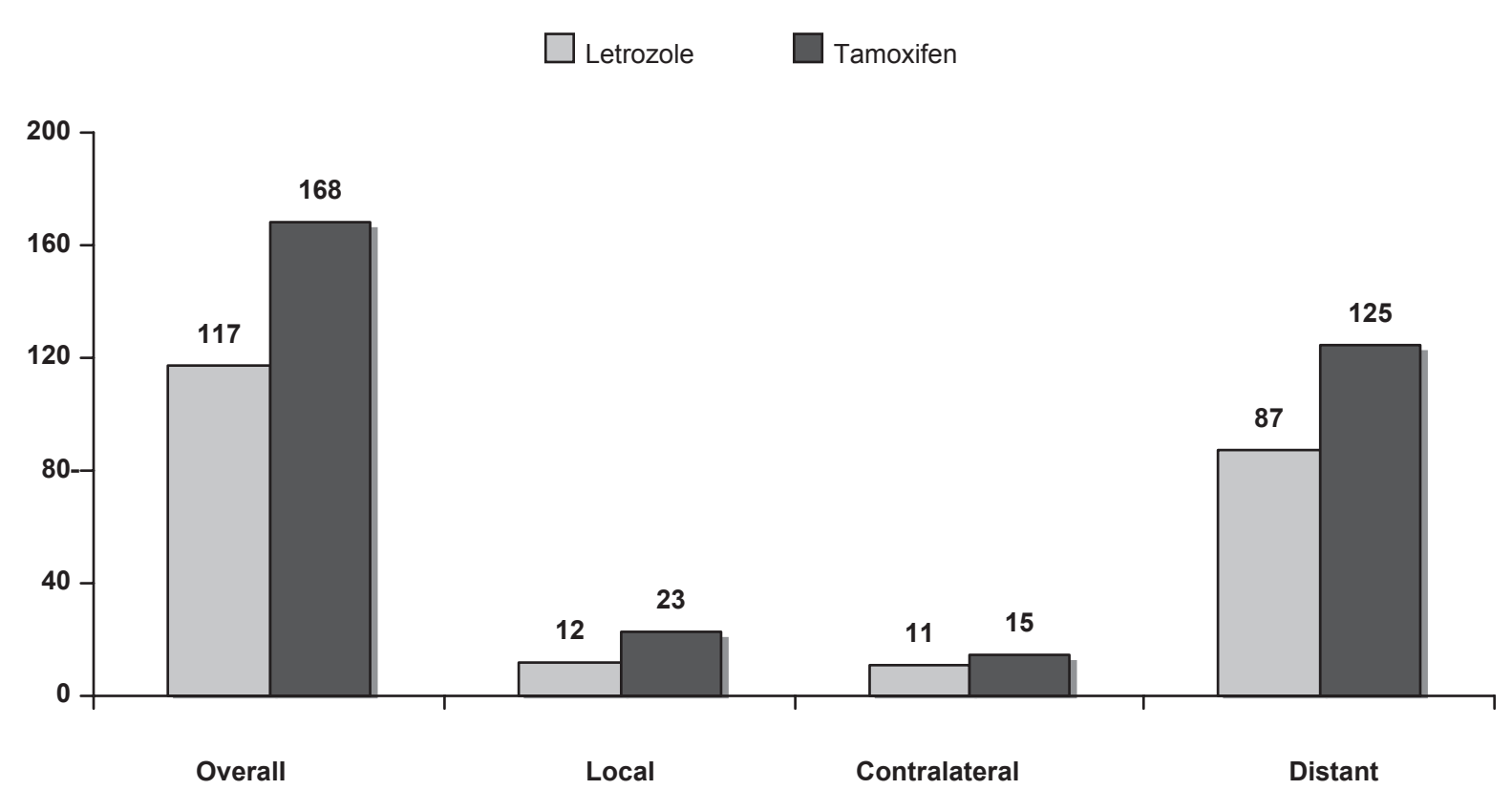

Figure 2 Reduction in overall, local, contralateral, and distant metastatic recurrences with letrozole over tamoxifen at early ( 2 years) follow-up in the Breast International Group I-98 trial. The corresponding reductions in each type of recurrence are $30.4 \%, 47.8 \%, 26.7 \%$, and $30.4 \%$, respectively. Drawn from data of Mauriac et al $2007 .{ }^{19}$ 
A

Letrozole $\square$ Anastrozole

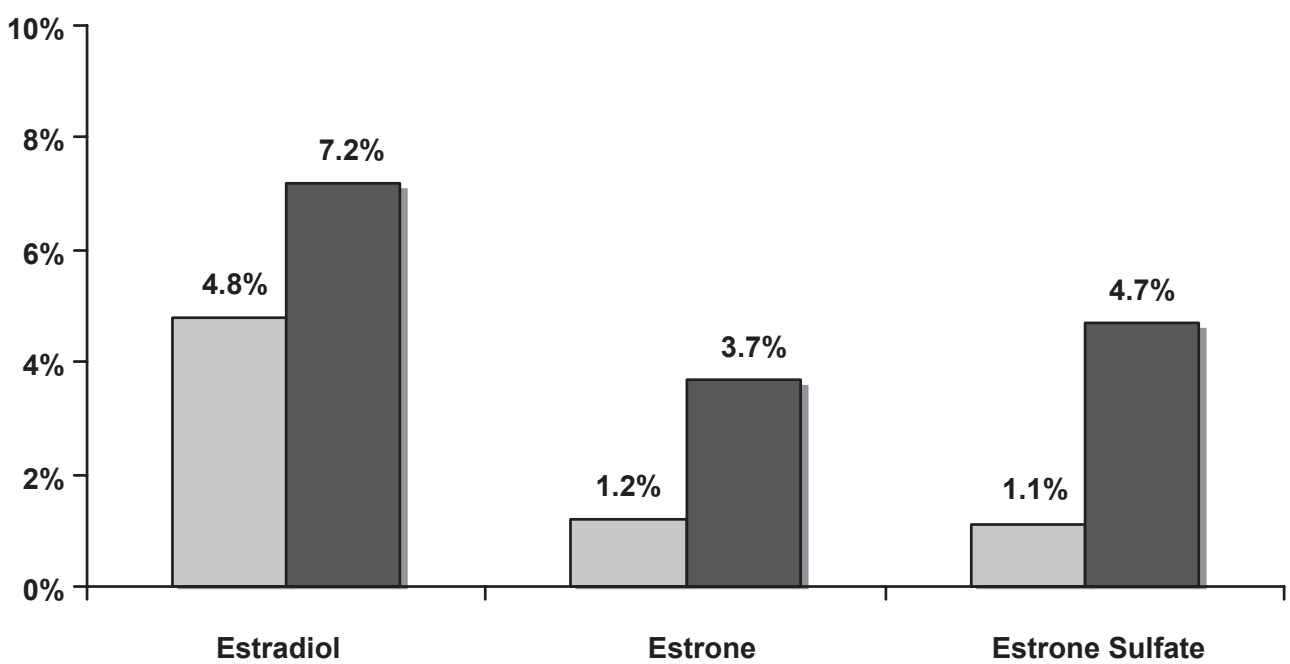

B

Letrozole $\square$ Anastrozole

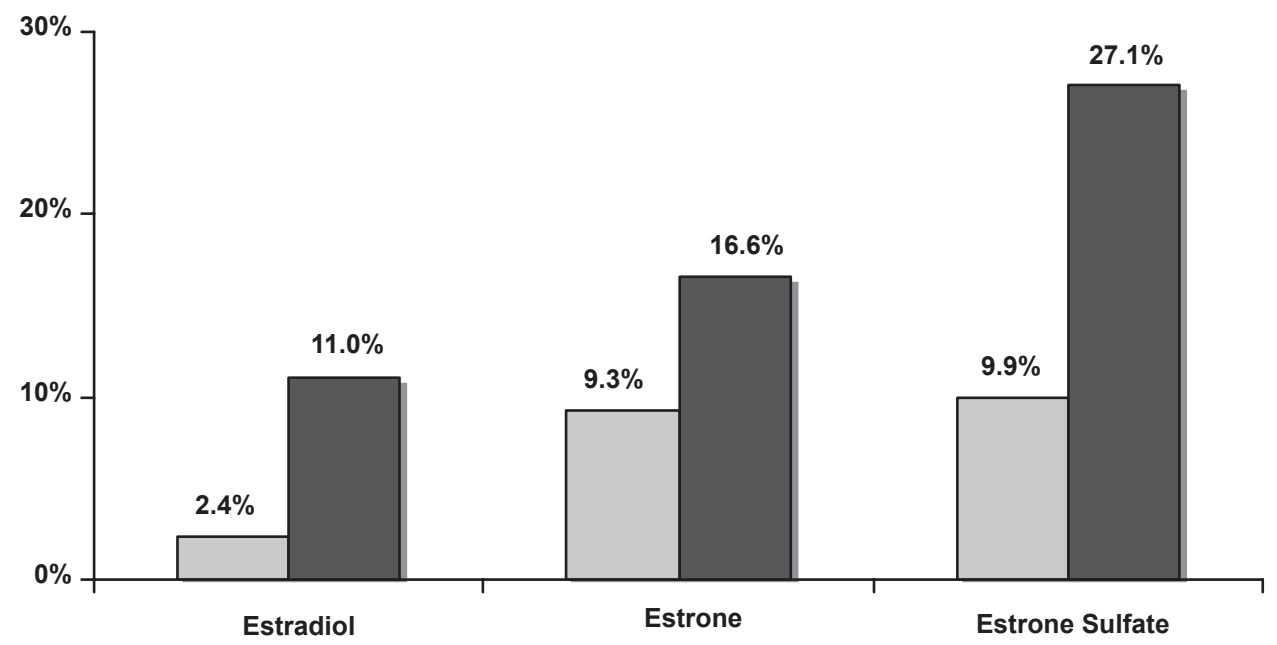

Figure 3 (A) Comparison of residual estrogen fractions in plasma following 6 weeks' treatment with letrozole or anastrozole. The difference in suppression was significant for each fraction (for estradiol, $P=0.018$; for estrone, $P=0.003$; for estrone sulfate, $P=0.003) .{ }^{16}(\mathbf{B})$ Comparison of residual estrogen fractions in tumor tissue following 16 weeks' neoadjuvant treatment with letrozole or anastrozole ( $P$ values not reported). Drawn from data of Geisler et al $2008 .{ }^{16}$

ARNO 95, and ITA) have examined switching to an AI (exemestane or anastrozole) after 2 to 3 years of prior tamoxifen in relation to continued tamoxifen, only patients remaining recurrence-free after the tamoxifen treatment were randomized, and events were considered only from the time of the switch onward..$^{8,10,11}$ The sequential therapy analysis of BIG 1-98, by comparison, considered all events from the time of randomization onward, allowing for a more accurate assessment of sequential treatment. ${ }^{5}$ Although the trial was not powered to compare efficacy of the sequential arms with letrozole monotherapy, this was believed to be the most relevant comparison, given the already proven superiority of letrozole over tamoxifen. In addition, comparisons with the tamoxifen monotherapy arms would not be interpretable because of the aforementioned crossover of patients from this arm to letrozole. The results of pairwise comparisons of the sequential arms with the letrozole monotherapy arm at a median follow-up of 71 months have been recently reported. ${ }^{5}$ As these comparisons were not a primary analysis, 2-sided 99\% confidence intervals (CIs) were presented, and as a 
superiority trial, the lack of significance cannot be inferred as equivalence between the treatments. Overall results did not show a significant benefit of either sequential approach in terms of DFS, TTDR, or OS (Table 2). Importantly, the risk of DM was consistently less with letrozole monotherapy compared with either sequential approach (Table 2). ${ }^{5}$ These initial findings support the importance of initiating with letrozole after surgery and maintaining letrozole therapy for 5 years to provide maximal benefit.

\section{Extended adjuvant therapy - MA.I 7}

Given the established superiority of AIs over tamoxifen, it is evident that AI therapy should be a component of adjuvant hormonal therapy, and this is not limited to the first 5 years after surgery; indeed, recurrence risk can extend beyond 5 years, even among relatively low-risk, $\mathrm{N}$ - patients. ${ }^{26}$ Results from the MA.17 trial have shown that adjuvant AI therapy with letrozole can be offered to those women who have already received 5 years of tamoxifen. This trial randomized patients with 4.5 to 6.0 years of prior adjuvant tamoxifen to further therapy with letrozole or placebo. ${ }^{12}$ Initial findings of this trial showed a significant, $43 \%$ reduction in recurrences by 2.4 years of follow-up, and this important finding resulted in a decision to unblind the trial and allow women on placebo the opportunity to cross over to letrozole. ${ }^{12}$ At a median 30-month follow-up, there was a significant $42 \%$ reduction in recurrence, a $37 \%$ reduction in contralateral breast cancers (HR $0.63 ; P=0.12$ ), and a significant $40 \%$ reduction in distant recurrence with letrozole over placebo (Table 1). ${ }^{13}$ While the observed $18 \%$ improvement in OS with letrozole over placebo was not significant in the overall population (Table 1), subgroup analysis showed a significant, $39 \%$ improvement in OS with letrozole in the $\mathrm{N}+$ subgroup ( $\mathrm{N}=2,360$; HR $0.61 ; P=0.04) .{ }^{13}$ A subsequent modeling study from MA.17 found a significant benefit of letrozole therapy with longer treatment, such that the longer patients

Table 2 Efficacy end points (hazard radio [HR] and $99 \%$ confidence interval $[\mathrm{Cl}]$ ) from the sequential therapy analysis of the Breast International Group (BIG) I-98 trial (pairwise comparisons with letrozole [LET] monotherapy) at a median 7I-month follow-up

\begin{tabular}{|c|c|c|c|c|}
\hline \multirow[t]{2}{*}{ End point } & \multicolumn{2}{|c|}{ TAM $\rightarrow$ LET vs LET } & \multicolumn{2}{|c|}{ LET $\rightarrow$ TAM vs LE } \\
\hline & HR & $99 \% \mathrm{Cl}$ & HR & $99 \% \mathrm{Cl}$ \\
\hline DFS & 1.05 & $0.84-1.32$ & 0.96 & $0.76-1.21$ \\
\hline OS & 1.13 & $0.83-1.53$ & 0.90 & $0.65-1.24$ \\
\hline TTDR & 1.22 & $0.88-1.69$ & 1.05 & $0.75-1.47$ \\
\hline
\end{tabular}

Drawn from data of Mouridsen 2009. ${ }^{5}$

Abbreviations: DFS, disease-free survival; OS, overall survival; TAM, tamoxifen, TTDR, time to distant recurrence. were exposed to letrozole, the greater the benefit. ${ }^{27}$ In the overall population, the improvement in DFS (6 months: HR 0.59, 48 months: HR 0.19; $P<0.0001$ ) and distant disease-free survival (DDFS; 6 months: HR 0.51, 48 months: HR $0.21 ; P=0.0013$ ) both significantly increased over time, whereas the improvement in OS remained similar (6 months: HR 0.87, 48 months: HR 0.79; $P=0.33) .{ }^{27}$ This suggests that, while the MA.17 trial was unblinded at a median of 30 months, the benefit of letrozole in DFS and DDFS extends well beyond this, up to 48 months.

Further analysis of MA.17 has examined outcomes in women who elected to cross over to letrozole following unblinding. A total of 1,579 of 2,383 patients (66\%) originally assigned to placebo elected to cross over to letrozole after unblinding. At a median of 64 months' follow-up, patients who received letrozole continued to show a significant 32\% improvement in DFS (HR 0.68; $P=0.0001$ ) and a $20 \%$ improvement in DDFS (HR 0.80; $P=0.082$ ), despite the crossover of these patients. ${ }^{28}$ Finally, another study has compared outcomes in patients who crossed over from placebo to letrozole (placebo $\rightarrow$ letrozole; $N=1,579$ ) with those who elected to remain on placebo following unblinding of the trial (placebo $\rightarrow$ placebo; $N=804$ ), allowing for an assessment of the efficacy of extended adjuvant letrozole in patients who had been off tamoxifen therapy for a median of 2.8 years (range, 1.1 to 7.1 years). ${ }^{29}$ The results of this analysis showed significant improvements in DFS (63\%; HR 0.37; $P<0.0001)$, DDFS (61\%; HR $0.39 ; P=0.004)$, and $\mathrm{OS}(70 \%$; HR $0.30 ; P<0.0001)$ for patients in the placebo $\rightarrow$ letrozole compared with the placebo $\rightarrow$ placebo group, suggesting a benefit of extended adjuvant letrozole over placebo, even for patients who had been off tamoxifen as long as 7 years. ${ }^{29}$ The results of MA. 17 demonstrate the efficacy of extended adjuvant letrozole and allow an important treatment option for patients that can provide longer protection against the risk of breast cancer recurrence.

\section{Safety profile of letrozole}

As adjuvant hormonal therapy is indicated for all women with $\mathrm{HR}+\mathrm{EBC}$, the assessment of AI safety has been, unfortunately, confounded by comparisons with treatment groups receiving tamoxifen, which can have beneficial effects on lipids, bone, and cardiovascular outcomes in PMW. ${ }^{14,30-34}$ Tamoxifen can also influence safety outcomes by virtue of its own AE profile, which includes an increased risk for endometrial abnormalities and/or cancers, thromboembolic events, and stroke. ${ }^{14,15}$ As AI therapy is associated with 
profound suppression of plasma estrogens, safety findings from the major AI trials have shown increases in osteoporosis and/or fracture risk, as well as musculoskeletal symptoms such as arthralgias and myalgias (commonly observed during menopause) in women receiving adjuvant AI therapy relative to tamoxifen. ${ }^{2,3,6,8,9}$ Unlike other AIs, the toxicity profile of letrozole has been studied in double-blind studies not only in relation to tamoxifen (BIG 1-98) but also in relation to placebo (MA.17). This allows for a more complete assessment of safety issues in populations receiving adjuvant tamoxifen, as well as those who have completed their tamoxifen treatment. In particular, BIG 1-98, as noted earlier, allowed for a very comprehensive assessment of cardiovascular safety, and the effect of letrozole on lipids and bone health also has been well studied. Other symptoms associated with estrogen suppression, such as joint and/or musculoskeletal symptoms, hot flashes, ${ }^{35}$ gynecological effects, and changes in mood and/or cognition, are a source of concern for many patients on AIs or tamoxifen, ${ }^{36}$ and available data regarding the impact of letrozole on these parameters is also reviewed below.

\section{Cardiovascular health}

There has been much improvement in the treatment of $\mathrm{EBC}$, and with the current standard of care, breast cancer has become more of a chronic condition, meaning most patients will live for an extended period with their disease while remaining at risk for disease recurrence. Considering the mean age of patients enrolled in many AI trials is about 60 to 65 years, ${ }^{2,8,10,13}$ other competing causes of death, such as cardiovascular disease, will become evident, and this needs to be considered in the overall outcome of clinical trials. BIG 1-98, as already noted, allowed for the prospective collection and grading of AEs using CTC 2.0 criteria, and lifelong assessment of cardiovascular outcomes, and this enables a very thorough assessment of the impact of these competing causes of death on outcome.

\section{Letrozole vs tamoxifen}

At 25.8 months' median follow-up, BIG 1-98 reported a higher incidence of hypercholesterolemia for patients assigned to letrozole vs those assigned to tamoxifen (Table 3 ), but this likely reflected a cholesterol-lowering effect of tamoxifen; cholesterol levels remained unchanged with letrozole, decreasing by only $-1.8 \%$ at 24 months, whereas the reduction was $-14.1 \%$ by 24 months in tamoxifen-treated patients. $^{2}$ Hypercholesterolemia was reported at least once in more letrozole-treated vs tamoxifen-treated patients (Table 3), and most events were grade 1 (35.1\% and $17.3 \%$, respectively). Cholesterol assessments were prospectively collected every 6 months, and most were non-fasting. A single incident of elevated serum cholesterol was counted as an $\mathrm{AE}$ regardless of whether or not subsequent levels were normal, and as such, hypercholesterolemia events in BIG 1-98 cannot be considered a medical diagnosis. At 60.5 months, there was again more ( 2-fold), mostly grade 1 , hypercholesterolemia with letrozole, ${ }^{3}$ and thus, the results with regard to this $\mathrm{AE}$ have been consistent, with no evidence for an increasing risk for hypercholesterolemia with

Table 3 Cardiovascular safety: percent of patients with indicated event ( $P$ value) for patients in the letrozole (LET) versus tamoxifen (TAM) (Breast International Group [BIG] I-98) and LET versus placebo (PBO) groups (MA. I7), respectively 2,13

\begin{tabular}{|c|c|c|c|}
\hline \multirow[b]{3}{*}{ Follow-up } & \multicolumn{2}{|l|}{ BIG I-98 } & \multirow{2}{*}{$\frac{\text { MA. I } 7}{\text { LET vs PBO }}$} \\
\hline & \multicolumn{2}{|l|}{ LET vs TAM } & \\
\hline & $25.8 \mathrm{mo}$ & $60.5 \mathrm{mo}$ & $30.0 \mathrm{mo}$ \\
\hline $\mathbf{N}$ & 8,010 & 7,963 & 5,149 \\
\hline Hypertension & NR & NR & 5.0 vs $5.0(0.94)$ \\
\hline Hypercholesterolemia & 43.6 vs 19.2 (NR) & 48.7 vs $24 . I$ (NR) & 16.0 vs $16.0(0.79)$ \\
\hline CVA/TIA & I.0 vs $1.0(0.91)$ & I. 4 vs I.5 (NR) & 0.7 vs $0.6(\mathrm{NR})$ \\
\hline Thromboembolic event & I.5 vs $3.5(<0.00 \mathrm{I})$ & 2.0 vs 4.1 (NR) & 0.4 vs 0.2 (NR) \\
\hline Cardiovascular disease & NR & NR & 5.8 vs $5.6(0.76)$ \\
\hline Cardiac event & 4.1 vs $3.8(0.61)$ & 5.6 vs 5.4 (NR) & NR \\
\hline Ischemic heart disease & I.4 vs I.2 (0.28) & 2.I vs I.7 (NR) & NR \\
\hline Cardiac failure & 0.8 vs $0.4(0.01)$ & I.0 vs 0.8 (NR) & NR \\
\hline Other CV event & 0.5 vs $0.2(0.04)$ & 0.9 vs 0.5 (NR) & NR \\
\hline Grade 3-5 CV event & 3.7 vs $4.2(P=N S)$ & NR & NR \\
\hline Grade $3-5$ cardiac event & 2.1 vs I.I $(<0.00 \mathrm{I})$ & 2.8 vs I.7 (NR) & NR \\
\hline
\end{tabular}

Abbreviations: CVA/TIA, cerebrovascular accident/transient ischemic attack; NS, not significant; NR, not reported. 
letrozole over time (ie, 2.3 -fold at 25.8 months vs 2.0-fold at 60.5 months). In addition, if hypercholesterolemia were truly an effect of letrozole therapy, an increased overall cardiovascular risk would be expected over time, and, as detailed further below, this is not supported by long-term cardiovascular outcome data in BIG 1-98. The safety results of BIG 1-98 at 25.8 months and at 60.5 months showed a rare but higher incidence of grade 3-5 cardiac events with letrozole. However, over time, the cardiac safety findings are consistent, with no evidence of increasing risk for grade 3 to 5 cardiac events (Table 3 ) with letrozole in the large PCA population $(\mathrm{N}=8,010)$ of patients (ie, 1.9-fold higher at 25.8 months, 1.6-fold higher at 60.5 months).

Very similar findings were reported in a detailed safety analysis from BIG 1-98 $(\mathrm{N}=7,963)$ at a median 30.1-month follow-up. Total cholesterol decreased over time in both groups, but reduction was more pronounced in the tamoxifen group, while a decrease with letrozole was apparent after 30 months. ${ }^{37}$ More patients receiving letrozole had an increase from baseline in their total cholesterol at each follow-up visit, which explains the higher proportion of low-grade hypercholesterolemia, ${ }^{37}$ although in this analysis, almost all (91\%) of the measurements were non-fasting. Significantly more grade $3-5$ cardiac events $(2.4 \%$ vs $1.4 \% ; P=0.001)$, specifically grade 3 to 5 cardiac failure $(0.7 \%$ vs $0.3 \%$; $P=0.04$ ), were seen with letrozole, while significantly more grade 3 to 5 thromboembolic events ( $0.9 \%$ vs $2.3 \%$; $P<0.001)$ were seen with tamoxifen. ${ }^{37}$ This analysis also found that a prior cholesterol elevation was associated with reporting of a grade 3 to 5 cardiac $\mathrm{AE}$, but the association could not completely explain the increase in events with letrozole. Notably, the incidence (overall, grades 1 to 5) of ischemic heart disease $(1.7 \%$ vs $1.5 \% ; P=0.48)$, cerebrovascular events $(1.2 \%$ vs $1.2 \% ; P=0.92)$, and hypertension $(3.8 \%$ vs $3.4 \% ; P=0.37$ ) did not differ between the groups. ${ }^{37}$

Although also reportedly consistent with earlier results, updated, detailed safety findings from the monotherapy patients $(\mathrm{N}=4,922)$ at 76 months are not yet available. However, the number of deaths without a cancer event was identical with both letrozole and tamoxifen in this analysis, ${ }^{5}$ which argues against any significant adverse impact of letrozole on cardiac outcomes.

\section{Letrozole vs placebo - MA. I 7}

As tamoxifen is known to have beneficial effects on lipids and cardiac health, the comparison of letrozole with placebo in MA.17 allows for a more accurate assessment of the effects of letrozole on lipids and cardiovascular outcomes in a population of PMW who have all received prior tamoxifen. ${ }^{12}$ Analysis of safety in MA.17 did not show a significant difference in the incidence of hypercholesterolemia or cardiovascular disease between the letrozole and placebo treatment groups (Table 3). ${ }^{12,13}$ A companion lipid substudy of MA.17 has further examined lipid profiles over time (6, 12, 24, and 36 months) in letrozole- and placebo-treated patients $(\mathrm{N}=347)$. The overall conclusions of the study suggested no significant adverse impact of letrozole on serum cholesterol, high-density lipoprotein (HDL), low-density lipoprotein (LDL), or triglycerides in women without hyperlipidemia at baseline. ${ }^{38}$ The only significant differences in the percentage change from baseline between treatment groups were in HDL at 6 months $(P=0.049)$, LDL at 12 months $(P=0.033)$, and triglycerides at 24 months $(P=0.036) .{ }^{38}$ There was no significant difference between treatment groups in the percentage of patients exceeding predefined thresholds for lipid parameters. ${ }^{38}$

\section{Letrozole vs other Als}

The ATAC and BIG 1-98 trials differed in their collection of AEs, so cardiovascular safety results are not directly comparable. Nonetheless, increases in cholesterol and some cardiac outcomes are seen in patients with all AIs compared with tamoxifen. ${ }^{6,9,39}$ A recently reported comparative study of the lipid effects of letrozole and anastrozole suggests similar effects by these two nonsteroidal AIs on lipid parameters in PMW. ${ }^{40}$ In an open-label pharmacokinetic study (Anastrozole vs Letrozole, an Investigation of QUality Of life and Tolerability [ALIQUOT]), patients $(\mathrm{N}=57)$ were randomized to 12 weeks of letrozole followed by 12 weeks of anastrozole or the reverse sequence. There were no major differences between the drugs except for an increase in LDL cholesterol with letrozole at 6 months $(P=0.04)$ compared with anastrozole. Notably, in patients recently completing tamoxifen therapy, both drugs caused beneficial changes in lipid parameters (lower triglycerides) compared with those not having a prior tamoxifen exposure. ${ }^{40}$

\section{Summary - cardiovascular effects}

The overall results of BIG 1-98 and MA.17 do not suggest a significant adverse impact of letrozole therapy on hypercholesterolemia or cardiovascular outcomes. However, care should always be taken to monitor and appropriately treat patients with preexistent cardiovascular risk factors and/or hypercholesterolemia while undergoing therapy with letrozole or any AI. Although the number of events was rare, the increase in grade 3 to 5 cardiac AEs over tamoxifen in BIG 1-98 can only be partly explained by prior cholesterol 
elevation, and it has been suggested that the small increase in incidence was due to a vascular endothelial effect arising from the profound estrogen suppression by letrozole. ${ }^{37}$ The lack of such an effect in MA.17 suggests it is more likely due to the beneficial effect of tamoxifen on cardiac health. ${ }^{37}$ It should be noted as well that, despite any potential impact of either therapy on these outcomes in BIG 1-98, OS favors letrozole over the long term. In particular, as noted earlier, the number of deaths caused by a non-cancer event was identical in the monotherapy follow-up at 76 months, ${ }^{5}$ and similar findings were seen in the 60.5-month update of the PCA population. ${ }^{3}$ Long-term results from BIG 1-98 suggest no increase in the risk of cardiovascular events over time, despite the advance in age of women in BIG 1-98 from a median of 61 years at the start of the study ${ }^{2}$ to about $67+$ years by the latest follow-ups., ${ }^{3,5}$

\section{Bone health}

Declining estrogen levels are known to have an effect on bone health during natural menopause, and it can be expected that a profound further suppression of estrogen during AI therapy (Figure 3) in PMW will predispose women to loss of bone mineral density (BMD) and an accompanying increase in fracture risk.

\section{Letrozole vs tamoxifen - BIG I-98}

Safety findings of the PCA showed a significantly higher incidence of fracture (about 1.4-fold) with letrozole compared with tamoxifen, although these events were relatively infrequent, occurring in $<6 \%$ of patients (Table 4 ). A similar significantly higher incidence (about 1.3-fold) was seen in the 60.5-month PCA update, and again, the risk of fracture does not appear to be increasing over time (1.4-fold vs 1.3-fold).

\section{Letrozole vs placebo - MA.I7}

An effect of tamoxifen on bone health in the safety analysis of BIG 1-98 cannot be excluded, and thus, the comparison of letrozole with placebo in MA.17 may be more informative. New-onset osteoporosis was reported by more women on letrozole compared with placebo in MA.17, and the difference reached significance by 30 months of follow-up (Table 4). Importantly, fractures were again relatively infrequent $(<6 \%$ overall), and while trending higher with letrozole, the difference was not significant compared with placebo (Table 4).

\section{Letrozole vs other Als}

In a recent report from the ALIQUOT study, the impact of AI therapy with letrozole and anastrozole on bone turnover
Table 4 Bone and musculoskeletal events: percent of patients with indicated event ( $P$ value) for patients in the letrozole (LET) versus tamoxifen (TAM) (Breast International Group [BIG] I-98) and LET vs placebo (PBO) groups (MA.I7), respectively $2,3,13$

\begin{tabular}{lllll}
\hline & \multicolumn{1}{l}{ BIG I-98 } & & MA.I 7 \\
\cline { 2 - 3 } & LET vs TAM & & LET vs PBO \\
\hline Follow-up & $25.8 \mathrm{mo}$ & $60.5 \mathrm{mo}$ & $30.0 \mathrm{mo}$ \\
$\mathrm{N}$ & 8,010 & 7,963 & 5,149 \\
Osteoporosis & $\mathrm{NR}$ & $\mathrm{NR}$ & 8.1 vs $6.0(0.003)$ \\
Fracture & 5.7 vs $4.0(<0.00 \mathrm{I})$ & 7.5 vs $5.7(\mathrm{NR})$ & 5.3 vs $4.6(0.25)$ \\
\hline
\end{tabular}

Abbreviation: NR, not reported.

was compared. ${ }^{41}$ In this study, despite the above-noted greater suppression of estrogen with letrozole, both AIs had similar effects on bone, with an increase in bone turnover over time ( 6 months compared with 3 months). The findings of the study did not suggest any likely differential effect of either drug on fracture rate or the incidence of osteoporosis. Another important finding of the study was that both AIs caused significant increases in bone turnover when tamoxifen was withdrawn and replaced by an AI, suggesting that prior tamoxifen use has a major impact on AI-related bone effects. ${ }^{41}$ This was corroborated in another study that also found similar effects on bone turnover markers with the nonsteroidal AIs anastrozole and letrozole and the steroidal AI exemestane. ${ }^{42}$

\section{Treatment of bone loss - Z-FAST and ZO-FAST}

While the loss of BMD and the associated increase in fracture risk is an important safety consideration for patients receiving AI therapy, the efficacy benefits of AIs over tamoxifen in terms of reducing recurrences, especially DM, will likely outweigh this risk for a majority of patients. In addition, emerging evidence suggests that bisphosphonate therapy with zoledronic acid (ZA) can not only treat bone loss but also can effectively prevent AI-associated bone loss (AIBL). The efficacy and safety of concomitant bisphosphonate therapy with ZA to prevent AIBL in patients receiving letrozole has been investigated in two similarly designed trials, Z-FAST (United States) and ZO-FAST (Europe) (Zometa Femara Adjuvant Synergy Trial).These trials randomized patients receiving adjuvant letrozole to either upfront $Z A$, initiated at randomization and every 6 months, or delayed ZA, initiated with a post-baseline decrease of -2.0 in the T-score at either the lumbar spine or the total hip, or in the event of a non-traumatic clinical fracture. ${ }^{43,44}$ The 12-month results from the Z-FAST trial $(\mathrm{N}=602)$ showed that upfront ZA could effectively 
prevent bone loss at both the lumbar spine and total hip. Whereas BMD decreased in the delayed group, it increased in the upfront group, and the mean percent difference in BMD between the treatment groups was $4.4 \%$ at the lumbar spine $(P<0.0001)$ and $3.3 \%$ at the total hip $(P<0.0001)$. Moreover, nearly 4-fold more patients with normal BMD (at the lumbar spine and/or total hip) at baseline developed $\mathrm{mild} /$ moderate osteopenia by 12 months in the delayed group compared with the immediate group (12.6\% vs 3.4\%). Similarly, more patients with mild/moderate osteopenia at baseline progressed to severe osteopenia/osteoporosis by month 12 in the delayed group ( $14.8 \%$ vs $1.4 \%$, respectively). ${ }^{43}$ The incidence of low/no trauma fractures $(0.7 \%$ vs $1.0 \%)$, and the incidence of traumatic fractures ( $2 \%$ vs $2.3 \%)$, did not differ. At the 36-month follow-up, similar results were observed, with a difference at the lumbar spine of $6.7 \%(P<0.001)$ and at the total hip of $5.2 \%(P<0.001) .{ }^{45}$ The findings of Z-FAST thus demonstrate that AIBL in PMW with breast cancer receiving adjuvant letrozole can be effectively prevented with upfront ZA treatment, and bisphosphonate therapy represents an attractive option for patients who may be at risk for bone loss while on AIs. Recent results from the companion ZO-FAST study at 36 months are also suggestive of a further benefit of ZA therapy. In addition to preventing AIBL, as in Z-FAST, there was a significant improvement in DFS for patients receiving immediate ZA vs delayed ZA (HR 0.588; $P=0.0314) .{ }^{46}$ Reports of a beneficial effect of $\mathrm{ZA}$ treatment on disease recurrence in premenopausal women receiving hormonal therapy with goserelin and anastrozole in a different trial, ${ }^{47,48}$ and a similar emerging benefit in the Z-FAST trial, ${ }^{45}$ suggest that, in addition to its effective prevention of AIBL, upfront ZA therapy has an antitumor efficacy benefit as well.

\section{Musculoskeletal symptoms}

Musculoskeletal symptoms including arthralgia and myalgia are commonly seen during menopause and in women undergoing estrogen suppression. In BIG 1-98, the incidence of these events was higher with letrozole vs tamoxifen, but the difference was only significant in the case of arthralgia (Table 5). In MA.17, arthralgia and myalgia were observed in between $12 \%$ and $25 \%$ of the patients overall, and the incidence of both was significantly higher with letrozole relative to placebo (Table 5), which likely reflects the profound estrogen suppression in women on letrozole relative to placebo. Interestingly, recent data from the ATAC study have linked the emergence of joint symptoms such as arthralgia with the efficacy of AIs, such that patients displaying these symptoms have a lower risk for recurrence. ${ }^{49}$ Whether the same is true for letrozole will require further investigation, but these findings highlight the link between the estrogensuppressing activities of the AIs (as manifested by the occurrence of joint symptoms) and efficacy in terms of recurrence risk reduction. Evidence from recent studies also suggests that switching between AIs may be one way to alleviate joint symptoms in patients without losing the efficacy benefit of continued AI therapy. It had been reported previously that more than half of patients experiencing joint symptoms on one AI (letrozole or anastrozole) did not experience joint symptoms while on the other $\mathrm{AI} .{ }^{50}$ Findings from the Articular Tolerance of Letrozole (ATOLL) study $(\mathrm{N}=179$ ) examined the effect of a switch to letrozole in patients on anastrozole with joint pain severe enough to require discontinuation. At the end of 6 months, most (71.5\%) of the patients elected to remain on letrozole, while the remainder (28.5\%) discontinued because of severe joint pain. ${ }^{51}$ These findings support a simple yet effective treatment option (ie, switching from one AI to the other) for most patients with troublesome joint symptoms who might otherwise need to discontinue AI therapy.

\section{Cognitive function}

With the increased use of AIs as adjuvant hormonal therapy and the markedly greater suppression of plasma estrogens associated with such therapy, the effects of AI treatment on cognition will be important to consider. Studies in animals suggest that estrogen receptors in the brain can have an impact on cognition and/or cognitive performance, and that estrogens can have an important neuroprotective effect. ${ }^{52-54}$ Accordingly, the menopause-associated decline in estrogen level has been associated with declines in cognitive function, and conversely, some studies suggest that higher endogenous estrogens can prevent cognitive decline, although the data are not entirely consistent. ${ }^{52,55}$ Because cognitive decline can have a dramatic impact on quality of life (QOL) in long-term survivors of breast cancer, the potential impact of AI therapy needs further investigation. ${ }^{55}$

In a pilot study of 184 women enrolled in ATAC, the patient group receiving anastrozole or tamoxifen had significantly impaired verbal memory $(P=0.026)$ and processing speed $(P=0.032)$ relative to a healthy control group..$^{56,57}$ It should be noted that the ATAC study compared women with breast cancer to healthy controls, which can present biases, as the diagnosis of breast cancer alone can impact cognition. ${ }^{58}$ The Cognition in the Study of Tamoxifen and Raloxifene (Co-STAR) will be interesting, as the trial is 
Table 5 Other adverse events: percent of patients with indicated event ( $p$ value) for patients in the letrozole (LET) versus tamoxifen (TAM) (Breast International Group [BIG] I-98, Primary Core Analysis population) and LET versus placebo (PBO) groups (MA.I7), respectively $2,3,13$

\begin{tabular}{|c|c|c|c|}
\hline & BIG I-98 & & MA. 17 \\
\hline & LET vs TAM & & LET vs PBO \\
\hline Follow-up & $25.8 \mathrm{mo}$ & $60.5 \mathrm{mo}$ & $30.0 \mathrm{mo}$ \\
\hline$N$ & 8,010 & 7,963 & 5,149 \\
\hline Arthritis & $N R$ & $N R$ & 6.0 vs $5.0(0.07)$ \\
\hline Arthralgia & 20.3 vs I2.3 $(<0.00 \mathrm{I})$ & 21.9 vs 16.5 (NR) & 25.0 vs $21.0(<0.001)$ \\
\hline Myalgia & 6.4 vs $6.1(0.6 I)$ & 7.8 vs 7.4 (NR) & I5.0 vs I $2.0(0.004)$ \\
\hline Bone pain & $N R$ & NR & 5.0 vs $6.0(0.67)$ \\
\hline Vaginal bleeding & 3.3 vs $6.6(<0.001)$ & 3.9 vs 8.0 (NR) & 6.0 vs $8.0(0.005)$ \\
\hline Vaginal dryness & $N R$ & $N R$ & 6.0 vs $5.0(0.26)$ \\
\hline Hot flashes/flushes & 33.5 vs $38.0(<0.00 \mathrm{I})$ & 35.2 vs 39.5 (NR) & 58.0 vs $54.0(0.003)$ \\
\hline Night sweats/sweating & I3.9 vs I6.2(0.004) & I4.7 vs I6.9 (NR) & 30.0 vs $29.0(0.48)$ \\
\hline
\end{tabular}

Abbreviation: NR, not reported.

designed to compare the cognitive effects of the two agents in the absence of disease ${ }^{59}$ Another study $(\mathrm{N}=31)$ comparing women receiving anastrozole and tamoxifen in ATAC found that women receiving anastrozole had significantly poorer performance on learning and memory measures than women receiving tamoxifen. ${ }^{60}$ Although preliminary, the data are suggestive of a role for estrogen in cognition and a potential impact of AI therapy on some measures of cognitive function, and this is an area that is certainly worthy of additional investigation. Analyses looking into the effect of letrozole compared with tamoxifen on cognitive function have been conducted for BIG 1-98 and are to be presented at the 2009 American Society of Clinical Oncology meeting.

\section{Other events}

Menopausal symptoms such as hot flashes, and gynecologic events including vaginal discharge, bleeding, and/or dryness may also occur in a substantive proportion of women receiving adjuvant hormonal therapies with either AIs or tamoxifen, and these can be a source of distress and reduced QOL for women undergoing adjuvant therapy. One "patient-perspective" study reported hot flashes, weight gain, insomnia, and joint aches as most troublesome for patients on hormonal therapy, with all of these except joint aches occurring at approximately equivalent frequency in AI- and tamoxifen-treated patients. ${ }^{36}$ Other notable symptoms included loss of libido, vaginal dryness, vaginal discharge, muscle aches, and mood changes, all of which occurred at roughly similar frequencies in about $70 \%$ to $85 \%$ of the patients receiving tamoxifen or AIs. These findings illustrate the disparity between safety results from AI trials and "real life" situations and highlight the importance of addressing these concerns to assure patient compliance with hormonal therapy.

\section{Letrozole vs tamoxifen - BIG I-98}

Results from BIG 1-98 (Table 5) comparing initial adjuvant letrozole with tamoxifen have shown a significantly lower incidence of vaginal bleeding with letrozole, as well as a lower incidence of vasomotor events such as hot flashes and night sweats. However, hot flashes still occurred in about one third of the patients in each group, as would be expected in a population of PMW undergoing hormonal treatment (Table 5). There was also less need for endometrial biopsy in patients taking letrozole vs tamoxifen $(2.3 \%$ vs $9.1 \%$; $P<0.001)$ in the PCA, and the incidence of invasive endometrial cancer, although quite rare $(<0.5 \%)$ in both groups, trended lower with letrozole $(0.1 \%$ vs $0.3 \% ; P=0.18) .^{2}$ Long-term follow-up of the PCA population at 60.5 months of median follow-up has yielded very similar findings with respect to these events (Table 5). ${ }^{3}$

\section{Letrozole vs placebo - MA. 17}

The population in MA.17 included women who had received tamoxifen for 4.5 to 6.0 years, ${ }^{12}$ and the incidence of vasomotor symptoms at the latest follow-up was high in this patient group, with $50 \%$ or more reporting hot flashes, and more patients in the letrozole group reporting this symptom (Table 5). Night sweats were also seen in about one third of the patients, but the incidence was similar between the treatment groups. The incidence of vaginal dryness also 
was not significantly different between the groups; however, vaginal bleeding was significantly higher in the placebo group (Table 5). Although rare $(\leq 6 \%)$ in either group, other events that were significantly higher with letrozole vs placebo were alopecia ( $5 \%$ vs $3 \% ; P=0.01)$ and anorexia $(6 \%$ vs $4 \% ; P=0.039) .{ }^{13}$

\section{QOL considerations}

Studies evaluating the impact of AIs on QOL outcomes have generally suggested no overall adverse impact of AI therapy. Physical and mental QOL measures such as the 36-item short form health survey (SF-36), the estrogen suppression-related scale (Menopause-Specific Quality of Life [MENQOL]), and others have been assessed in QOL studies from MA.17, ATAC, and the IES. ${ }^{61-64}$ In the MA.17 QOL study, patients were stratified by age group ( $<65$ years and $\geq 65$ years), and no statistically significant differences (letrozole vs placebo) were seen in either age group in MENQOL psychosocial and physical domains or in the SF-36 mental QOL summary score, physical functioning, role-physical, general health, social functioning, role-emotional, and mental health subdomains. ${ }^{62}$ By comparison, both age groups showed significant differences in favor of placebo for SF-36 bodily pain and MENQOL vasomotor symptoms. Significant differences in favor of letrozole were seen for the MENQOL sexual functioning domain in the younger group. Differences in the older group favored placebo in the SF-36 physical summary score and vitality at selected time points. Nevertheless, although significant, the differences were not considered clinically meaningful by current methods. ${ }^{62}$ Another assessment of QOL from MA.17 showed no detrimental effect of extended adjuvant letrozole on overall QOL, despite small differences in QOL scores in selected domains such as physical function, bodily pain, vitality, vasomotor, and sexual. The findings were consistent with the letrozole toxicity profile in women with menopause relatedsymptoms (eg, arthralgias, hot flashes), which could be a source of decreased QOL for some patients receiving letrozole. ${ }^{61}$

\section{Conclusions}

Both BIG 1-98 and MA.17 have provided pivotal data regarding the safety and efficacy of letrozole as adjuvant hormonal therapy in PMW with EBC. The efficacy results of MA.17 suggest that, for PMW who have not had the benefit of initial AI therapy during the first 5 years after surgery and have already completed tamoxifen therapy, extended adjuvant therapy with letrozole offers significant benefits over no additional treatment, even when letrozole is started as many as 7 years after completion. ${ }^{13,29}$ Notably, however, the efficacy results of the sequential therapy analysis from BIG 1-98 illustrate that there is no better treatment strategy than upfront letrozole. ${ }^{5}$ Indeed, the results from BIG 1-98 have established the efficacy of initial adjuvant hormonal treatment with letrozole over tamoxifen for PMW with EBC. The findings of this important study demonstrate that letrozole is significantly more effective than tamoxifen in reducing recurrences, particularly in its pronounced impact on reducing the risk of DM that occur early (at 2 years), ${ }^{19}$ when recurrence risk is greatest. ${ }^{21}$ With longer follow-up, the OS benefit is emerging in BIG 1-98 (Table 1), and these findings further illustrate the importance of reducing early DM in improving survival for women with EBC.

The MA.17 trial also has provided valuable data on the safety profile of letrozole in relation to placebo, whereas BIG 1-98 and other large AI trials such as ATAC and IES have compared AI therapy with tamoxifen, which confounds the safety interpretation. The results of MA.17 suggest no adverse impact of letrozole therapy on hypercholesterolemia, or on the overall incidence of cardiovascular AEs compared with placebo, whereas the AE profile of letrozole in BIG 1-98, while similar to that of other AIs, may be subject to considerable "noise" by virtue of the comparison with tamoxifen. Nevertheless, long-term follow-up of BIG 1-98 does not suggest an increasing risk of these important AEs over time. In fact, as noted earlier, the overall difference between treatment groups in hypercholesterolemia and grade 3 to 5 cardiac AEs appears to diminish. In addition, although a detailed analysis of safety is pending, the long-term results of the monotherapy cohort show 40 fewer overall (cancer-related) deaths with letrozole, an identical incidence of noncancer-related deaths, and safety results that are consistent with the known AE profile of both agents. ${ }^{5}$ Also of considerable interest are the recent findings of the Z-FAST and ZO-FAST studies, which suggest not only that bisphosphonate therapy with ZA has a beneficial effect in preventing AIBL but also that such therapy may reduce recurrences (an unexpected outcome). ${ }^{46}$ Long-term findings of BIG 1-98 and MA.17 thus support the efficacy of adjuvant hormonal therapy with letrozole in PMW, with or without prior tamoxifen treatment, with a predictable and manageable toxicity profile expected for most patients.

\section{Acknowledgments and disclosures}

The author thanks Maria Soushko, PhD, Phase Five Communications Inc., New York, NY, for editorial assistance with this manuscript. Financial support for editorial assistance was provided by Novartis Oncology. 


\section{References}

1. Aiello EJ, Buist DS, Wagner EH, et al. Diffusion of aromatase inhibitors for breast cancer therapy between 1996 and 2003 in the Cancer Research Network. Breast Cancer Res Treat. 2008;107:397-403.

2. Thurlimann B, Keshaviah A, Coates AS, et al. Breast International Group (BIG) 1-98 Collaborative Group. A comparison of letrozole and TAMOXIFEN in postmenopausal women with early breast cancer. N Engl J Med. 2005;353:2747-2757.

3. Thurlimann B. Letrozole vs tamoxifen as adjuvant endocrine therapy for postmenopausal women with receptor-positive breast cancer. Update of the BIG 1-98 Primary Core Analysis (PCA). Presented at the 39th St. Gallen Symposium; May 7-9, 2009; St. Gallen, Switzerland. Abstract 0161

4. Coates AS, Keshaviah A, Thurlimann B, et al. Five years of letrozole compared with tamoxifen as initial adjuvant therapy for postmenopausal women with endocrine-responsive early breast cancer: update of study BIG 1-98. J Clin Oncol. 2007;25:486-492.

5. Mouridsen H; on behalf of the IBCSG BIG 1-98 trial group. Letrozole monotherapy vs tamoxifen monotherapy or vs letrozole in sequence with tamoxifen for postmenopausal women with endocrine-responsive early breast cancer. Cancer Res. 2009;69 Suppl 2:66s. Abstract 13.

6. Howell A, Cuzick J, Baum M, et al. ATAC Trialists' Group. Results of the ATAC (Arimidex, Tamoxifen, Alone or in Combination) trial after completion of 5 years' adjuvant treatment for breast cancer. Lancet. 2005;365:60-62.

7. Forbes JF, Cuzick J, Buzdar A, Howell A, Tobias JS, Baum M. Arimidex, Tamoxifen, Alone or in Combination (ATAC) Trialists' Group. Effect of anastrozole and tamoxifen as adjuvant treatment for early-stage breast cancer: 100-month analysis of the ATAC trial. Lancet Oncol. 2008;9:45-53.

8. Coombes RC, Hall E, Gibson LJ, et al. Intergroup Exemestane Study. A randomized trial of exemestane after two to three years of tamoxifen therapy in postmenopausal women with primary breast cancer. $N$ Engl J Med. 2004;350:1081-1092.

9. Coombes RC, Kilburn LS, Snowdon CF, et. al. Intergroup Exemestane Study. Survival and safety of exemestane versus tamoxifen after 2-3 years' tamoxifen treatment (Intergroup Exemestane Study): a randomised controlled trial. Lancet. 2007;369:559-570.

10. Kaufmann M, Jonat W, Hilfrich J, et al. Improved overall survival in postmenopausal women with early breast cancer after anastrozole initiated after treatment with tamoxifen compared with continued tamoxifen: the ARNO 95 Study. J Clin Oncol. 2007;25:2664-2670.

11. Boccardo F, Rubagotti A, Puntoni M, et al. Switching to anastrozole versus continued tamoxifen treatment of early breast cancer: preliminary results of the Italian Tamoxifen Anastrozole trial. J Clin Oncol. 2005;23:5138-5147.

12. Goss PE, Ingle JN, Martino S, et al. A randomized trial of letrozole in postmenopausal women after five years of tamoxifen therapy for early-stage breast cancer. N Engl J Med. 2003;349:1793-1802.

13. Goss PE, Ingle JN, Martino $\mathrm{S}$, et al. Randomized trial of letrozole following tamoxifen as extended adjuvant therapy in receptor-positive breast cancer: updated findings from NCIC CTG MA.17. J Natl Cancer Inst. 2005;97:1262-1271.

14. Braithwaite RS, Chlebowski RT, Lau J, George S, Hess R, Col NF. Meta-analysis of vascular and neoplastic events associated with tamoxifen. J Gen Intern Med. 2003;18:937-947.

15. Fisher B, Costantino JP, Wickerham DL, et al. Tamoxifen for prevention of breast cancer: report of the National Surgical Adjuvant Breast and Bowel Project P-1 Study. J Natl Cancer Inst. 1998;90: 1371-1388.

16. Geisler J, Helle H, Ekse D, et al. Letrozole is superior to anastrozole in suppressing breast cancer tissue and plasma estrogen levels. Clin Cancer Res. 2008;14:6330-6335.

17. Dixon JM, Renshaw L, Young O, et al. Letrozole suppresses plasma estradiol and estrone sulphate more completely than anastrozole in postmenopausal women with breast cancer. J Clin Oncol. 2008;26:1671-1676.
18. Rossi E, Morabito A, Di Rella F, et al. Endocrine effects of adjuvant letrozole compared with tamoxifen in hormone-responsive postmenopausal patients with early breast cancer: The HOBOE Trial. J Clin Oncol. 20 April 2009 [Epub ahead of print]. 10.1200/ JCO.2008.18.6213. Available at: http://jco.ascopubs.org/cgi/ doi/10.1200/JCO.2008.18.6213.

19. Mauriac L, Keshaviah A, Debled M, et al. BIG 1-98 Collaborative Group; International Breast Cancer Study Group. Predictors of early relapse in postmenopausal women with hormone receptorpositive breast cancer in the BIG 1-98 trial. Ann Oncol. 2007;18: 859-867.

20. Lamerato S, Havstad S, Gandhi D, Jones R. Chlebowski. Breast cancer recurrence and related mortality in US pts with early breast cancer. J Clin Oncol. 2005;23(16S):62s. Abstract 738.

21. Mansell J, Monypenny IJ, Skene AI, et al. Patterns and predictors of early recurrence in postmenopausal women with estrogen receptor-positive early breast cancer. Breast Cancer Res Treat. 2008 Dec 27. [Epub ahead of print].

22. Houghton J. Initial adjuvant therapy with anastrozole (A) reduces rates of early breast cancer recurrence and adverse events compared with tamoxifen (T) - data reported on behalf of the ATAC ('Arimidex', tamoxifen, alone or in Combination) Trialists' group. Ann Oncol. 2006;17 Suppl 9:ix94. Abstract 243PD.

23. Rugo HS. Adjuvant therapies: distant disease-free survival as a predictor of overall survival. Presented at: Primary Therapy of Early Breast Cancer 2007: 10th International Conference; March 14-17, 2007; St. Gallen, Switzerland. Abstract 120.

24. Monnier A. Refining the postmenopausal breast cancer treatment paradigm: the FACE trial. Expert Rev Anticancer Ther. 2006 6:1355-1359.

25. O'Shaughnessy J. A decade of letrozole: FACE. Breast Cancer Res Treat. 2007;105 Suppl 1:67-74.

26. Hortobagyi GN, Kau SW, Buzdar AU, et al. What is the prognosis of patients with operable breast cancer (BC) five years after diagnosis? Proc Am Soc Clin Oncol. 2004;22(14S):Abstract 585.

27. Ingle JN, Tu D, Pater JL, et al. Duration of letrozole treatment and outcomes in the placebo-controlled NCIC CTG MA.17 extended adjuvant therapy trial. Breast Cancer Res Treat. 2006;99:295-300.

28. Ingle $\mathrm{JN}, \mathrm{Tu} \mathrm{D}$, Pater $\mathrm{JL}$, et al. Intent-to-treat analysis of the placebo-controlled trial of letrozole for extended adjuvant therapy in early breast cancer: NCIC CTG MA.17. Ann Oncol. 2008; 19:877-882.

29. Goss PE, Ingle JN, Pater JL, et al. Late extended adjuvant treatment with letrozole improves outcome in women with early-stage breast cancer who complete 5 years of tamoxifen. J Clin Oncol. 2008;26: 1948-1955. Erratum in: J Clin Oncol. 2008;26:3659.

30. Cuzick J, Allen D, Baum M, et al. Long term effects of tamoxifen. Biological effects of Tamoxifen Working Party. Eur J Cancer. 1992; 29A:15-21.

31. Love RR, Mazess RB, Barden HS, et al. Effects of TAMOXIFEN on bone mineral density in postmenopausal women with breast cancer. N Engl J Med 1992;326:852-856.

32. Kristensen B, Ejlertsen B, Dalgaard P, et al. Tamoxifen and bone metabolism in postmenopausal low-risk breast cancer patients: a randomized study. J Clin Oncol. 1994;12:992-997.

33. McDonald CC, Alexander FE, Whyte BW, Forrest AP, Stewart HJ. Cardiac and vascular morbidity in women receiving adjuvant TAMOXIFEN for breast cancer in a randomised trial. The Scottish Cancer Trials Breast Group. BMJ. 1995;311:977-980.

34. Monnier A. The effects of adjuvant aromatase inhibitor therapy on lipid profiles. Expert Rev Anticancer Ther. 2006;6:1653-1662.

35. Monnier A. Clinical management of adverse events in adjuvant therapy for hormone-responsive early breast cancer. Ann Oncol. 2007; 18 Suppl 8:viii 36-44.

36. Garreau JR, Delamelena T, Walts D, Karamlou K, Johnson N. Side effects of aromatase inhibitors versus tamoxifen: the patients' perspective. Am J Surg. 2006;192:496-498. 
37. Mouridsen H, Keshaviah A, Coates AS, et al. Cardiovascular adverse events during adjuvant endocrine therapy for early breast cancer using letrozole or tamoxifen: safety analysis of BIG 1-98 trial. J Clin Oncol. 2007;25:5715-5722.

38. Wasan KM, Goss PE, Pritchard PH, et al. The influence of letrozole on serum lipid concentrations in postmenopausal women with primary breast cancer who have completed 5 years of adjuvant TAMOXIFEN (NCIC CTG MA.17L). Ann Oncol. 2005;16:707-715.

39. Buzdar A, Howell A, Cuzick J, et al. Arimidex, Tamoxifen, Alone or in Combination Trialists' Group. Comprehensive side-effect profile of anastrozole and tamoxifen as adjuvant treatment for early-stage breast cancer: long-term safety analysis of the ATAC trial. Lancet Oncol. 2006;7:633-643.

40. McCaig F, Renshaw L, Williams L, et al. A randomised study comparing the effects of anastrozole and letrozole on lipid metabolism. Cancer Res. 2009;69 Suppl 2:150s-151s. Abstract 1152.

41. McCaig F, Renshaw L, Williams L, et al. A randomised study of the effects of letrozole and anastrozole on bone turnover. Breast Cancer Res Treat. 2007;106 Suppl 1:S109. Abstract 2074.

42. McCaig F, Renshaw L, Williams L, et al. A randomized study of the effects of anastrozole (A), letrozole (L), and exemestane (E) on bone. Cancer Res. 2009;69 Suppl 2:148s. Abstract 1145.

43. Brufsky A, Harker WG, Beck JT, et al. Zoledronic acid inhibits adjuvant letrozole-induced bone loss in postmenopausal women with early breast cancer. J Clin Oncol. 2007;25:829-836.

44. Bundred NJ, Campbell ID, Davidson N, et al. Effective inhibition of aromatase inhibitor-associated bone loss by zoledronic acid in postmenopausal women with early breast cancer receiving adjuvant letrozole: ZO-FAST study results. Cancer. 2008;112:1001-1010.

45. Brufsky A, Bosserman L, Caradonna R, et al. The effect of zoledronic acid on aromatase inhibitor-associated bone loss in postmenopausal women with early breast cancer receiving adjuvant letrozole: the Z-FAST study 36-month follow-up. Breast Cancer Res Treat. 2007;106 Suppl 1:S8. Abstract 27.

46. Eidtmann H, Bundred N, DeBoer R, et al. The Effect of Zoledronic Acid on Aromatase Inhibitor Associated Bone Loss in Postmenopausal Women with Early Breast Cancer Receiving Letrozole: 36 Months Follow-up of ZO-FAST. Cancer Res. 2009;69 Suppl 2:74s. Abstract 44.

47. Gnant MF, Mlineritsch B, Luschin-Ebengreuth G, et al. Zoledronic Acid prevents cancer treatment-induced bone loss in premenopausal women receiving adjuvant endocrine therapy for hormone-responsive breast cancer: a report from the Austrian Breast and Colorectal Cancer Study Group. J Clin Oncol. 2007;25:820-828.

48. Gnant MB, Mlineritsch W, Schippinger G, et al. Adjuvant ovarian suppression combined with tamoxifen or anastrozole, alone or in combination with zoledronic acid, in premenopausal women with hormoneresponsive, stage I and II breast cancer: First efficacy results from ABCSG-12. J Clin Oncol. 2008:26 Suppl:1006s. Abstract LBA4.

49. Cuzick J, Sestak I, Cella D, et al. ATAC Trialists' Group. Treatmentemergent endocrine symptoms and the risk of breast cancer recurrence: a retrospective analysis of the ATAC trial. Lancet Oncol. 2008;9: $1143-1148$
50. Renshaw L, McHugh RGN, Williams L, et al. Comparison of joint problems reported by patients in a randomized adjuvant trial of anastrozole and letrozole. Breast Can Res Treat. 2007;106 Suppl 1: S108-S109. Abstract 2072.

51. Briot K, Bastit L, Rotarsky M, et al. Effects of switching aromatase inhibitors on arthralgia: the ATOLL study. Cancer Res. 2008; 69 Suppl:147s-148s. Abstract 1142.

52. Markou A, Duka T, Prelevic GM. Estrogens and brain function. Hormones (Athens). 2005;4:9-17.

53. Spencer JL, Waters EM, Romeo RD, Wood GE, Milner TA, McEwen BS. Uncovering the mechanisms of estrogen effects on hippocampal function. Front Neuroendocrinol. 2008;29:219-237.

54. Singh M, Dykens JA, Simpkins JW. Novel mechanisms for estrogen-induced neuroprotection. Exp Biol Med (Maywood). 2006; 231:514-521.

55. Rugo HS, Ahles T. The impact of adjuvant therapy for breast cancer on cognitive function: current evidence and directions for research. Semin Oncol. 2003;30:749-762.

56. Shilling V, Jenkins V, Fallowfield L, Howell T. The effects of hormone therapy on cognition in breast cancer. J Steroid Biochem Mol Biol. 2003;86:405-412.

57. Jenkins V, Shilling V, Fallowfield L, Howell A, Hutton S. Does hormone therapy for the treatment of breast cancer have a detrimental effect on memory and cognition? A pilot study. Psychooncology. 2004;13:61-66.

58. Debess J, Riis JØ, Pedersen L, Ewertz M. Cognitive function and quality of life after surgery for early breast cancer in North Jutland, Denmark. Acta Oncol. 2009;48:532-540.

59. ClinicalTrials.gov Identifier: NCT00687102. Effects of selective estrogen receptor modulators on cognitive aging: cognition in the study of tamoxifen and raloxifene. Available from: http://clinicaltrials. gov/ct2/show/NCT00687102.

60. Bender CM, Sereika SM, Brufsky AM, et al. Memory impairments with adjuvant anastrozole versus tamoxifen in women with early-stage breast cancer. Menopause. 2007;14:995-998.

61. Whelan TJ, Goss PE, Ingle JN, et al. Assessment of quality of life in MA.17: a randomized, placebo-controlled trial of letrozole after 5 years of tamoxifen in postmenopausal women. J Clin Oncol. 2005;23:6931-6940.

62. Abetz L, Brandman J, Barghout V, de la Loge C, Arbuckle R. No differences in quality of life for letrozole relative to placebo in postmenopausal women with early breast cancer regardless of age: results from the MA-17 study. Eur J Cancer Suppl. 2005;3:96. Abstract 342.

63. Fallowfield LJ, Bliss JM, Porter LS, et al. Quality of life in the intergroup exemestane study: a randomized trial of exemestane versus continued tamoxifen after 2 to 3 years of tamoxifen in postmenopausal women with primary breast cancer. J Clin Oncol. 2006;24: 910-917.

64. Cella D, Fallowfield L, Barker P, Cuzick J, Locker G, Howell A; ATAC Trialists' Group. Quality of life of postmenopausal women in the ATAC ("Arimidex", tamoxifen, alone or in combination) trial after completion of 5 years' adjuvant treatment for early breast cancer. Breast Cancer Res Treat. 2006;100:273-284.
Therapeutics and Clinical Risk Management

\section{Publish your work in this journal}

Therapeutics and Clinical Risk Management is an international, peerreviewed journal of clinical therapeutics and risk management, focusing on concise rapid reporting of clinical studies in all therapeutic areas, outcomes, safety, and programs for the effective, safe, and sustained use of medicines. This journal is indexed on PubMed Central, CAS,

\section{Dovepress}

EMBase, Scopus and the Elsevier Bibliographic databases. The manuscript management system is completely online and includes a very quick and fair peer-review system, which is all easy to use. Visit http://www.dovepress.com/testimonials.php to read real quotes from published authors. 